\title{
Diagnostic Difficulties Encountered in Milky Serum of a 4 Year Old Baby with Severe Diabetic Ketoacidosis and Hypertriglyceridemia Causing Acute Pancreatitis
}

\author{
Neeru Gupta*, Dinesh yadav, Chhaya Gupta \\ Sri Balaji Action Medical Institute, Paschim vihar, New Delhi, India
}

\begin{abstract}
Milky/ frothy serum samples can be received sporadically in cases of severe hypertriglyceridemia which can pose diagnostic challenge for the routine processing and result generation. We report difficulties encountered in processing of such sample in undiagnosed and comatosed 4 year old girl of new onset type 1 diabetes mellitus, severe diabetic ketoacidosis and hypertriglyceridemia induced acute pancreatitis. She had markedly elevated serum triglycerides $[13,846 \mathrm{mg} / \mathrm{dL}]$ with high cholesterol [1267 mg/dl], serum amylase [150 U/L], serum lipase [230 U/L], $\mathrm{pH} 6.85$ and bicarbonate $6 \mathrm{mEq} / \mathrm{L}$. Arterial blood gas analysis and urine chemistry were performed for the initial diagnosis. After establishing the diagnosis she was managed with insulin therapy and conservative management.
\end{abstract}

Keywords: Diabetes Mellitus Type 1, Hypertriglyceridaemia, Diabetic Ketoacidosis, Acute Pancreatitis, Lipemic Sample.

\section{Introduction}

Diabetic ketoacidosis [DKA] is an acute metabolic complication reflective of extremes of abnormal insulin homeostasis mainly in type 1 diabetes mellitus [T1DM] ${ }^{1}$. Acute pancreatitis, peripheral venous thrombosis, pulmonary oedema, severe hypertriglyceridaemia [HTG] and cerebral oedema are rare complications found in diabetic ketoacidosis patient. Severe hypertriglyceridaemia can increase risk of acute pancreatitis, especially in patients with triglycerides levels higher than $1,000-1,722 \mathrm{mg} / \mathrm{dl}^{2}$ Prevalence of severe hypertriglyceridaemia was found in about $8 \%$ of adults with diabetic ketoacidosis, ${ }^{3}$ but few data have been reported in children with severity ranging from asymptomatic to severe acute pancreatitis.

We report a case of four (4) year old girl child presenting with new onset Type 1 diabetes mellitus with severe diabetic ketoacidosis, severe hyperlipidemia and acute pancreatitis. The objective of this report is to present and describe the clinical features, laboratory investigations with difficulties faced in the processing of milky sample, case management and natural course of hypertriglyceridaemia in the child.

\section{Case Presentation}

A 4 year old female presented in the emergency with complaints of polyuria and polydipsia for past one month increasing significantly for last fifteen (15) days. The child had lost weight. For last two days she had developed gradual and progressive breathing difficulty along with lethargy and become unconscious in the morning. She was a normal full term delivered child and vaccinated for age with no past suggestive history or family history of diabetes. Her diet was normal. On examination she had heart rate of $152 / \mathrm{min}$, weak pulse, cold peripheries, respiratory rate of 52/ min, severely dehydrated, normal temperature, no cyanosis, clear chest, normal heart sounds with no signs of meningeal irritation on CNS examination. On laboratory investigations Haemoglobin - $24.7 \mathrm{gm} \%$ [lipemic sample], Packed cell volume $-29 \%$, Total leucocyte count $-33,300$ $\mathrm{cu} / \mathrm{mm}$, Differential leucocyte count - polymorphous 67, Lymphocytes - 33, Total platelet count -2.8 lakh cu/ $\mathrm{mm}$, Blood urea $-18 \mathrm{mg} / \mathrm{dl}$, serum creatinine $-0.6 \mathrm{mg} /$ dl, , C-reactive protein $-2.1 \mathrm{mg} / \mathrm{dl}$. Her blood sample was milky for initial 4 to 5 days (Figure 1). Biochemical parameters done sequentially from day 1 till day 28 are depicted in the table 1. The child was admitted in PICU and immediately resuscitated with normal saline boluses. A diagnosis of new onset type, diabetes mellitus with severe diabetic ketoacidosis was made and child was started on injectable insulin, deficit correction and injectable antibodies. Arterial line was secured in right femoral artery. Her sensorium, general condition and metabolic acidosis gradually improved and diabetic ketoacidosis resolved over 36 hours. USG abdomen and amylase / lipase done suggested acute pancreatitis. Child was continued on injectable insulin and IV fluids for hyperlipidemia and pancreatitis. Her serum TG and cholesterol gradually came down. Child was started on oral feeding and subcutaneous insulin and started on oral feeding once she became stable and asymptomatic. The child was discharged on injectable insulin advised RBC monitoring charting and repeats TG, cholesterol, lipase / amylase after four days. 


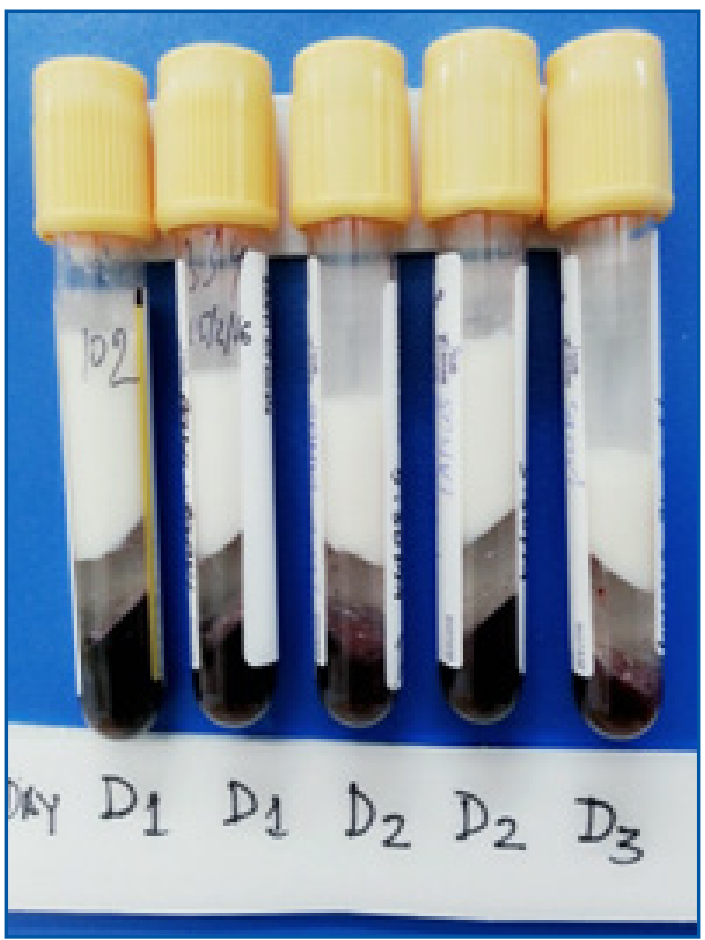

Fig. 1: Milky/frothy serum sample from day 1 to day 3 .

Table 1: Laboratory investigations (sequential)

\begin{tabular}{|c|c|c|c|c|c|c|c|c|c|c|c|}
\hline \multicolumn{12}{|c|}{ Sequential Investigations } \\
\hline & Day-1 & After $16 \mathrm{hr}$ & Day-2 & Day-3 & Day-4 & Day-5 & Day-6 & Day-8 & Day-14 & Day-28 & Bio Ref Range \\
\hline Triglyceride, mg/dL & 13846 & & 11147 & 5609 & 3455 & 2210 & 1573 & 675 & 490 & 90 & $<150$ \\
\hline Total Cholesterol, mg/dL & 1267 & & 1037 & 929 & 710 & & 660 & 555 & 395 & 110 & $<200$ \\
\hline $\mathrm{HDL}-\mathrm{C}, \mathrm{mg} / \mathrm{dL}$ & 64 & & 56 & 47 & & & & & & & $40-60$ \\
\hline Amylase, U/L & 75 & & 150 & 90 & 40 & & & 100 & 135 & 60 & $28-100$ \\
\hline Lipase, U/L & 120 & & 230 & 240 & 110 & & & 442 & 133 & 45 & $13-60$ \\
\hline Glucose, mg/dL & 451 & 229 & 237 & 236 & 179 & & & & & & $60-100$ \\
\hline $\mathrm{pH}$ & 6.85 & & 7.15 & 7.36 & 7.43 & & & & & & $7.35-7.45$ \\
\hline Bicarbonate, mEq/L & 6 & 9 & 11 & 18.4 & 19.2 & & & & & & $22-28$ \\
\hline Sodium, mEq/L & 114 & 124 & 129 & 135 & & & & & & & $135-148$ \\
\hline Potassium, mEq/L & 3.9 & 2.3 & 2.5 & 2.4 & & & & & & & $3.5-5.3$ \\
\hline Chloride, $\mathrm{mEq} / \mathrm{L}$ & 138 & & 136 & 138 & & & & & & & $98-107$ \\
\hline Ca ionised, mg/dL & 4.01 & 4.73 & 5.29 & & & & & & & & $4.6-5.4$ \\
\hline $\mathrm{HbA} 1 \mathrm{c}, \%$ & 7.8 & & & & & & & & & & $4.8-5.9$ \\
\hline Urine glucose, mg/dL & 100 & & & & & & & & & & Nil \\
\hline Urine ketones, $\mathrm{mg} / \mathrm{dL}$ & 50 & & & & & & & & & & Nil \\
\hline Apolipoprotein (Apo A1), mg/dL & & & & & & & & & 84 & & $105-205$ \\
\hline Apolipoprotein (Apo B), mg/dL & & & & & & & & & 301 & & $55-130$ \\
\hline Apo B/ Apo A1 Ratio & & & & & & & & & 3.58 & & $0.35-0.98$ \\
\hline Lipoprotein (a); Lp(a) mg/dL & & & & & & & & & 20.1 & & $<30$ \\
\hline Cardio CRP, mg/L & & & & & & & & & 0.9 & & $<1.0$ \\
\hline
\end{tabular}




\begin{tabular}{|l|l|l|l|l|l|l|l|l|l|l|l|}
\hline & \multicolumn{7}{|c|}{ Sequential Investigations } & & & & \\
\hline C-Peptide, ng/mL & Day-1 & After 16hr & Day-2 & Day-3 & Day-4 & Day-5 & Day-6 & Day-8 & Day-14 & Day-28 Bio Ref Range \\
\hline Lipoprotein Electrophoresis & & & & & & & & & & & $0.81-3.85$ \\
\hline HDL, \% & & & & & & & & & & & \\
\hline LDL, \% & & & & & & & & & 27.7 & & $42.3-69.5$ \\
\hline VLDL, \% & & & & & & & & & 70.1 & & $2.0-31.2$ \\
\hline Chylomicrons & & & & & & & & & Present & & Nil \\
\hline LDL Cholesterol, mg/dL & & & & & & & & & 271 & & $<110$ \\
\hline
\end{tabular}

\section{Discussion}

Severe hypertriglyceridaemia from insulin deficiency causing pancreatitis in new onset type 1 diabetes mellitus is a rare but serious complication of diabetic ketoacidosis in children ${ }^{4}$. Diagnosis is based on lipemic appearance of serum caking of chylomicrons on serum on refrigeration, serum triglycerides in excess of $1000 \mathrm{mg} / \mathrm{dL} .{ }^{5}$ Early diagnosis is important to prevent complications such as acute and chronic pancreatitis and pancreatic necrosis. Arterial blood gas analysis results rarely influence emergency physicians decisions on diagnosis, treatment or disposition in suspected DKA as stated in study by Ma et $\mathrm{al}^{6}$, however in our patient because of technical difficulties in processing highly lipemic (milky and frothy) sample for routine requested parameters in analyzer, diagnosis of diabetic ketoacidosis was established by results of $\mathrm{ABG}$ (pH-6.85, bicarbonate- $6 \mathrm{mEq} / \mathrm{L}$, glucose- $451 \mathrm{mg} / \mathrm{dL}$ ) and presence of urinary glucose/ ketones which actually helped the treating clinician to formulate the initial treatment protocol. Frier et $\mathrm{al}^{7}$ suggested that if serum triglyceride concentration exceeds $2,500 \mathrm{mg} / \mathrm{dl}$ measured electrolytes can decrease by over $5 \%$ because of the intracellular movement of serum lipid components. Similarly in our case decrease sodium levels upto $114 \mathrm{mEq} / \mathrm{L}$ was noted which was managed conservatively and gradually corrected. Nair et $\mathrm{al}^{3}$ observed that the specificity of elevated amylase (At three times the normal level) was $97 \%$ for diagnosing acute pancreatitis while the specificity of elevated lipase (At the same level) was only $91 \%$ indicate high amylase as a critical marker of acute pancreatitis. Normoamylasemia is possible in about $50 \%$ of patients with hypertriglyceridaemia induced pancreatitis. The mechanism is believed to be the interference with in vitro determination of the actual amylase level by disturbance of the calorimetric method. Serial dilutions of the sample could reduce interference of light transmission by hyperlipidemic serum ${ }^{8,9}$. In our patient we also faced similar interference which was resolved by serial dilutions following manufacturer's instructions. Kota et al ${ }^{1}$ also observed elevated lipase and amylase levels as in our patient. Abdominal ultrasound is a useful tool in supporting the diagnosis of acute pancreatitis. Pancreatic enlargement and hypoechogenicity on ultrasound is virtually diagnostic of acute pancreatitis ${ }^{10}$. In our case also acute pancreatitis diagnosis was supported by abdominal ultrasonography. In the present case hypertriglyceridaemia was controlled with insulin without lipid lowering agents and patient is kept on regular follow up with periodic testing for triglycerides, cholesterol, lipase, amylase and random blood sugar monitoring charting. However in severe hypertriglyceridaemia, physicians should consider the application of plasma exchange to avoid complications.

\section{Conclusion}

Technical difficulties are encountered in processing highly lipemic (milky/frothy) samples for routine requested parameters in comatosed, undiagnosed cases of severe hyperglycemia with hypertriglyceridemia. Performance of arterial blood gas analysis and urinary glucose/ ketones can be used as the two parameters which can resolve the issues as in this case. Expertise of Laboratory physician in handling such cases by selecting the suitable and output generating parameters is the need of the day to help the clinician in diagnosing and management of such cases.

\section{References}

1. Kota SK, Jammula S, Kota SK, Mehra LK and Modi KD. Acute pancreatitis in association with diabetic ketoacidosis in a newly diagnosed type 1 diabetes mellitus patient: case based review. International Journal of Clinical cases and investigations 2012; 4(1): $56-60$

2. Saengkaew T, Sahaki trungruang $\mathrm{T}$, Wacharasindhu $\mathrm{S}$, Supornsilchai V. DKA with severe hypertriglyceridaemia and cerebral edema in an adolescent boy: A case study and review of the literature. Case report in Endocrinology 2016; e 4

3. Nair S, Yadav D, Pitchumoni C.S. Association of diabetic ketoacidosis and acute pancreatitis: obervation in 100 consecutive episodes of diabetic ketoacidosis.American J of Gastoentrology 2000; 95(10): 2795 - 2800.

4. Wolfgram PM,MacDonald MJ. Severehypertriglyceridaemia causing acute pancreatitis in a child with new onset type 1 diabetes mellitus presenting in ketoacidosis. J Pediatr Intensive care 2013; 2(2): $77-80$. 
5. Chaurasiya OS, Kumar L, Sethi RS .An infant with milky blood: An unusual but treatable case of familial hyperlipidemia. Indian J Biochem 2013: 28(2): 206 - 209.

6. Ma OJ, Rush MD, Godfrey MM, Gaddis G. 2003. Arterial blood gas results rarely influence emergency physician management of patients with suspected diabetic ketoacidosis. ACAD Emerg Med 2013; 10(8): 836 - 841.

7. Frier BM, Steer CR, Baird JD, Bloomfield S. Misleading plasma electrolytes in diabetic children with severe hyperlipidemia. Arch Dis Child. 1980: 55; 771 - 775.
8. ,Yadav D,Nair S ,NorkusEP, Pitchumoni CS, Nonspecific hyperamylaesemia and hyperlipasemia in diabetic ketoacidosis:incidence and correlation with biochemical abnormalities. Am J Gastroenterol. 2000; 95: 3123 - 3128.

9. Hahn SK, Park JH, Lee JH, Lee JK,Kim KA.Severe hypertriglyceridemia in diabetic ketoacidosis accompanied by acute pancreatitis : Case report.J Korean Med Sci 2010;25(9):1375-1378

10. 10) Van Sonnenberg E, P Tehunmoni CS. Prolonged hyperamylasemia in diabetic ketoacidosis. JAMA 1976; 236: $482-483$.

*Corresponding author:

Dr.Neeru Gupta, BN-41,East Shalimar Bagh,Delhi.110088 INDIA

Phone: +91 9871603553

Email: drguptaneeru@gmail.com

Financial or other Competing Interests: None. 